Pacific Journal of Mathematics

THE SPLITTING OF OPERATOR ALGEBRAS 


\section{THE SPLITTING OF OPERATOR ALGEBRAS}

\section{Sze-KaI Tsui aNd Steve Wright}

We say the singly generated $C^{*}$-algebra, $C^{*}\left(T_{1} \oplus T_{2}\right)$, splits if $C^{*}\left(T_{1} \oplus T_{2}\right)=C^{*}\left(T_{1}\right) \oplus C^{*}\left(T_{2}\right)$. A necessary and sufficient condition is derived for the splitting of $C *\left(T_{1} \oplus T_{2}\right)$ in terms of the topological structure of the primitive ideal space of $C^{*}\left(T_{1} \oplus T_{2}\right)$. In particular, when $C^{*}\left(T_{1} \oplus T_{2}\right)$ is strongly amenable, the necessary and sufficient condition can be simplified and does not depend on the topology of the primitive ideal space of $C^{*}\left(T_{1} \oplus T_{2}\right)$. Several applications of this theorem, such as the cases, among others, where $T_{1}$, $T_{2}$ are compact operators, and $C^{*}\left(T_{1}\right), C^{*}\left(T_{2}\right)$ have only finite-dimensional irreducible representations, are discussed. For the splitting of the $W^{*}$-algebra, $W^{*}\left(T_{1} \oplus T_{2}\right)$, two equivalent conditions are derived which are quite different in nature. It is also shown that $W^{*}\left(T_{1} \oplus T_{2}\right)$ splits if either $W^{*}\left(\operatorname{Re} T_{1} \oplus \operatorname{Re} T_{2}\right)$ or $W *\left(\operatorname{Im} T_{1} \oplus \operatorname{Im} T_{2}\right)$ splits, but the converse is false. An example is given to show that $W^{*}\left(T_{1} \oplus T_{2}\right)$ splits whereas $C^{*}\left(T_{1} \oplus T_{2}\right)$ does not.

1. Introduction. Let $\mathscr{A}$ be a $C^{*}$-algebra. If $\mathscr{A}$ has an identity element and $T$ is in $\mathscr{A}, C^{*}(T)$ will denote the $C^{*}$-subalgebra of $\mathscr{A}$ generated by $T$ and the identity element; if $\mathscr{A}$ has no identity element, $C^{*}(T)$ will denote the $C^{*}$-subalgebra of $\mathscr{T}$ generated by $T$ alone. If $\mathscr{B}$ is another $C^{*}$-algebra and $\mathscr{A} \oplus \mathscr{B}$ is the $C^{*}$-direct sum of $\mathscr{A}$ and $\mathscr{B}$, one can ask the following question: Given $T_{1} \oplus T_{2}$ in $\mathscr{A} \oplus \mathscr{B}$, when does $C^{*}\left(T_{1} \oplus T_{2}\right)=C^{*}\left(T_{1}\right) \oplus C^{*}\left(T_{2}\right)$ ? One always has $C^{*}\left(T_{1} \oplus T_{2}\right) \leqq C^{*}\left(T_{1}\right) \oplus C^{*}\left(T_{2}\right)$, and if equality holds, we say $C^{*}\left(T_{1} \oplus T_{2}\right)$ splits. A similar question can be posed in the context of $W^{*}$-algebras. Given $W^{*}$-algebras $\mathscr{R}, \mathscr{S}$ and $T_{1} \oplus T_{2}$ in $\mathscr{R} \oplus \mathscr{S}$, when does $W^{*}\left(T_{1} \oplus T_{2}\right)=W^{*}\left(T_{1}\right) \oplus W^{*}\left(T_{2}\right)\left(W^{*}(T)=\right.$ the $W^{*}$-algebra generated by $\left.T\right)$ ? As in the $C^{*}$-algebra case, $W^{*}\left(T_{1} \oplus T_{2}\right)$ is said to split if equality holds.

In this paper, necessary and sufficient conditions are derived for the splitting of $C^{*}\left(T_{1} \oplus T_{2}\right)$ and $W^{*}\left(T_{1} \oplus T_{2}\right)$. These results should be compared with theorems in [2], [7], [5], and [6], where the splitting problem for various functors involving the direct sum is treated. Indeed, the results in the present paper can be viewed as "self-adjoint" analogs of the non-self-adjoint situations of this previous work.

Acknowledgment. The authors express their appreciation to John B. Conway for bringing the splitting problem for operator alge- 
bras to their attention. We also wish to thank Erik Christensen for pointing out an error in an early formulation of Theorem 3.4.

2. The splitting of $C^{*}\left(T_{1} \oplus T_{2}\right)$. If $\mathscr{A}$ is a $C^{*}$-algebra, Prim (A) will denote the primitive ideal space of $\mathscr{A}$ equipped with the hull-kernel topology, and $\operatorname{Irr}(\mathscr{A})$ will stand for the set of all irreducible representations of $\mathscr{A}$. If $E$ is a central projection in $\mathscr{A}$ and $\pi$ is a representation of $\mathscr{A}, \pi_{E}$ is the representation of $\mathscr{A}$ defined by $\pi_{E}(T)=\pi(T E)(T \in A)$.

We denote by $\mathscr{C}(\mathscr{A})$ the multiplier algebra of $\mathscr{A} ; \mathscr{C}(\mathscr{A})$ can be characterized as the largest $C^{*}$-subalgebra of $\mathscr{A}^{* *}$, the enveloping von Neumann algebra of $\mathscr{A}$, which contains $\mathscr{A}$ as a closed, two-sided ideal. If $\pi$ is in $\operatorname{Irr}(\mathscr{A})$, then $\pi^{\prime}$ denotes the unique extension of $\pi$ to an irreducible representation of $\mathscr{H}(\mathscr{A})$ (since $\mathscr{A}$ is a closed, two-sided ideal of $\mathscr{C}(\mathscr{A}), \pi^{\prime}$ exists for each $\pi$ in $\operatorname{Irr}(\mathscr{A}))$.

We begin by stating a noncommutative $C^{*}$-algebra analog of the Silov idempotent theorem ([15], Theorem 8.6). Its proof is obtained from a straightforward application of the Dauns-Hofmann theorem ([10], Theorem 3; [13] Corollary 4.7), and is therefore left to the reader.

Proposition. Let $\left\{\Sigma_{1}, \Sigma_{2}\right\}$ be a disconnection of $\operatorname{Prim}(\mathscr{A})$, $\mathscr{A}$ a $C^{*}$-algebra. Then there exists a unique central projection $E$ of $\mathscr{M}(\mathscr{A})$ such that

$$
\begin{aligned}
& \Sigma_{1}=\left\{\operatorname{ker} \pi: \pi \in \operatorname{Irr}(\mathscr{A}), \pi^{\prime}=\pi_{E}^{\prime}\right\}, \\
& \Sigma_{2}=\left\{\operatorname{ker} \pi: \pi \in \operatorname{Irr}(\mathscr{C}), \pi^{\prime}=\pi_{I-E}^{\prime}\right\} .
\end{aligned}
$$

Conversely, any nontrivial central projection $E$ of $\mathscr{C}(\mathscr{A})$ induces a disconnection of $\operatorname{Prim}(\mathscr{A})$ in this way.

Now let $\mathscr{A}_{i}, i=1,2$, be $C^{*}$-algebras and let $\pi_{i}$ be a representation of $\mathscr{A}_{i}, i=1,2$. We define a representation $\tilde{\pi}_{i}$ of $\mathscr{A}_{1} \oplus \mathscr{A}_{2}$ by "evaluation at coordinates", i.e.,

$$
\tilde{\pi}_{i}: A_{1} \oplus A_{2} \longrightarrow \pi_{i}\left(A_{i}\right) \quad\left(A_{1} \oplus A_{2} \in \mathscr{A}_{1} \oplus \mathscr{A _ { 2 }}\right) .
$$

In particular, if $T_{1} \oplus T_{2}$ is a fixed element in $\mathscr{A}_{1} \oplus \mathscr{A}_{2}$ and $\sigma \epsilon$ $\operatorname{Irr}\left(C^{*}\left(T_{i}\right)\right), i=1,2$, then $\tilde{\sigma}$ is an irreducible representation of $C^{*}\left(T_{1} \oplus T_{2}\right)$. With this in mind, we now state and prove the main theorem of this section.

TheOREM 2.1. Let $\mathscr{B}_{i}, i=1,2$, be $C^{*}$-algebras with $T_{1} \oplus T_{2}$ a fixed element in $\mathscr{B}_{1} \oplus \mathscr{B}_{2}$. Then $C^{*}\left(T_{1} \oplus T_{2}\right)$ splits if and only if 
the sets

$$
\Sigma_{i}=\left\{\operatorname{ker} \widetilde{\sigma}_{i}: \sigma_{i} \in \operatorname{Irr}\left(C^{*}\left(T_{i}\right)\right)\right\}, \quad i=1,2,
$$

disconnect $\operatorname{Prim}\left(C^{*}\left(T_{1} \oplus T_{2}\right)\right)$.

Proof. $\quad \Longrightarrow)$. Let $\mathscr{A}=C^{*}\left(T_{1}\right) \oplus C^{*}\left(T_{2}\right), \quad \mathscr{A}_{i}=C^{*}\left(T_{i}\right), i=1,2$. Since $\mathscr{A}^{* *}=\mathscr{A}_{1}^{* *} \oplus \mathscr{A}_{2}^{* *}$, there exist orthogonal central projections $E_{1}, E_{2}$ in $\mathscr{A}^{* *}$ with $I=E_{1}+E_{2}, E_{1}=I \oplus 0, E_{2}=0 \oplus I$. Thus $E_{i} \mathscr{A} \subseteq \mathscr{A}, i=1,2$. Since, for $\pi$ in $\operatorname{Irr}(\mathscr{A}), \pi^{\prime}=\pi_{E_{1}}^{\prime} \Leftrightarrow \pi$ vanishes on $0 \oplus \mathscr{A}_{2} \Leftrightarrow \pi=\widetilde{\sigma}$ for some $\sigma$ in $\operatorname{Irr}\left(\mathscr{A}_{1}\right)$, we conclude that $\Sigma_{1}=$ $\left\{\operatorname{ker} \pi: \pi \in \operatorname{Irr}(\mathscr{A}), \pi^{\prime}=\pi_{E_{1}}^{\prime}\right\}$, and similarly $\Sigma_{2}=\{\operatorname{ker} \pi: \pi \in \operatorname{Irr}(\mathscr{A})$, $\left.\pi^{\prime}=\pi_{1-E_{1}}^{\prime}\right\}$. By the previous proposition we have that $\left\{\Sigma_{1}, \Sigma_{2}\right\}$ disconnects Prim( $\mathscr{A})$.

$(\Longleftrightarrow)$. Let $\mathscr{A}=C^{*}\left(T_{1} \oplus T_{2}\right)$ and $\mathscr{\mathscr { A } _ { i }}=C^{*}\left(T_{i}\right)$ for $i=1,2$. Due to the above proposition, there exists a central projection $E$ of $\mathscr{L}(\mathscr{A})$ such that

$$
\begin{aligned}
& \Sigma_{1}=\left\{\operatorname{ker} \pi: \pi \in \operatorname{Irr}(\mathscr{A}), \pi^{\prime}=\pi_{E}^{\prime}\right\} \\
& \Sigma_{2}=\left\{\operatorname{ker} \pi: \pi \in \operatorname{Irr}(\mathscr{A}), \pi^{\prime}=\pi_{1-E}^{\prime}\right\} .
\end{aligned}
$$

Let $A_{1} \oplus A_{2}$ be a fixed element in $\mathscr{A}$, and $\sigma$ in $\operatorname{Irr}\left(\mathscr{A}_{1}\right)$. By (2.1) there exists $\pi$ in $\operatorname{Irr}(\mathscr{A})$ such that $\operatorname{ker} \pi=\operatorname{ker} \tilde{\sigma}, \pi=\pi_{E}^{\prime}$. Thus $1-E$ is in $\operatorname{ker} \pi^{\prime}$, and so

$$
0=\pi^{\prime}\left((1-E)\left(A_{1}+A_{2}\right)\right)=\pi\left((1-E)\left(A_{1}+A_{2}\right)\right) .
$$

Hence $(1-E)\left(A_{1} \oplus A_{2}\right) \in \operatorname{ker} \pi=\operatorname{ker} \widetilde{\sigma}$, i.e.,

$$
\begin{aligned}
0 & =\tilde{\sigma}\left((1-E)\left(A_{1} \oplus A_{2}\right)\right) \\
& =\sigma\left(\left[(1-E)\left(A_{1} \oplus A_{2}\right)\right]_{1}\right) .
\end{aligned}
$$

Since $\sigma$ is arbitrary in $\operatorname{Irr}\left(\mathscr{A}_{1}\right)$ and $\operatorname{Irr}\left(\mathscr{A}_{1}\right)$ separates points of $\mathscr{\mathscr { A } _ { 1 }}$, we conclude that

$$
0=\left[(1-E)\left(A_{1} \oplus A_{2}\right)\right]_{1}
$$

Similarly

$$
0=\left[E\left(A_{1} \oplus A_{2}\right)\right]_{2}
$$

Thus,

$$
\begin{aligned}
E\left(A_{1} \oplus A_{2}\right) & =\left[E\left(A_{1} \oplus A_{2}\right)\right]_{2} \oplus 0 \\
(1-E)\left(A_{1} \oplus A_{2}\right) & =0 \oplus\left[(1-E)\left(A_{1} \oplus A_{2}\right)\right]_{2} .
\end{aligned}
$$

Adding (2.5) and (2.6) yields

$$
A_{1} \oplus A_{2}=\left[E\left(A_{1} \oplus A_{2}\right)\right]_{1} \oplus\left[(1-E)\left(A_{1} \oplus A_{2}\right)\right]_{2} .
$$


Hence

$$
A_{1}=\left[E\left(A_{1} \oplus A_{2}\right)\right]_{1}, \quad A_{2}=\left[(1-E)\left(A_{1} \oplus A_{2}\right)\right]_{2},
$$

whence by (2.5) and (2.6),

$$
E\left(A_{1} \oplus A_{2}\right)=A_{1} \oplus 0, \quad(1-E)\left(A_{1} \oplus A_{2}\right)=0 \oplus A_{2} .
$$

Since $E$ multiplies $\mathscr{A}, A_{1} \oplus 0$ and $0 \oplus A_{2}$ are both in $\mathscr{A}$. It follows that $C^{*}\left(T_{1} \oplus T_{2}\right)$ splits.

Let $T$ be a normal element of a $C^{*}$-algebra, $\mathscr{C}$. We identify the spectrum $\Lambda(T)$ with $\operatorname{Prim}\left(C^{*}(T)\right)$, if $\mathscr{A}$ has an identity element. It is easy to see that $\Lambda\left(T_{1} \oplus T_{2}\right)=\Lambda\left(T_{1}\right) \cup \Lambda\left(T_{2}\right)$ for $T_{1}, T_{2}$ in $\mathscr{A}$. We therefore deduce from Theorem 2.1:

Corollary 2.2. Let $T_{1}$ and $T_{2}$ be normal elements in a $C^{*}$-algebra $\mathscr{A}$. If . has an identity, then $C^{*}\left(T_{1} \oplus T_{2}\right)$ splits if and only if $\Lambda\left(T_{1}\right) \cap \Lambda\left(T_{2}\right)=\varnothing$. If . has no identity, then, $C^{*}\left(T_{1} \oplus T_{2}\right)$ splits if and only if $\Lambda\left(T_{1}\right) \cap \Lambda\left(T_{2}\right)=\{0\}$.

Of particular interest is the case $\mathscr{A}_{1}=\mathscr{A}_{2}=\mathscr{B}(\mathscr{C})$, where $\mathscr{B}(\mathscr{Y})$ denotes the $C^{*}$-algebra of all bounded operators on the Hilbert space $\mathscr{C}$. The following results indicate the utility of Theorem 2.1.

COROLLARY 2.3. Suppose $T_{1}$ and $T_{2}$ are irreducible operators on $\mathscr{Y}$. Then $C^{*}\left(T_{1} \oplus T_{2}\right)$ splits if and only if $\operatorname{Prim}\left(C^{*}\left(T_{1} \oplus T_{2}\right)\right)$ is disconnected.

Proof. $I \oplus 0$ and $0 \oplus I$ are the only possible nontrivial central projections in $C^{*}\left(T_{1} \oplus T_{2}\right)$. If $\operatorname{Prim}\left(C^{*}\left(T_{1} \oplus T_{2}\right)\right)$ is disconnected, we hence conclude by the proposition preceeding Theorem 2.1 that $C^{*}\left(T_{1} \oplus T_{2}\right)$ contains $I \oplus 0$, and therefore splits.

Suppose that $T_{1}$ and $T_{2}$ are isometries on $\mathscr{C}$ with von NeumannWold decompositions $T_{\imath}=U_{i} \oplus S_{i}, i=1,2$, i.e., $U_{i}$ is unitary and $S_{i}$ is a (possibly trivial) unilateral shift. If either $S_{1}$ or $S_{2}$ is nonzero, it follows from [4] that $C^{*}\left(T_{1} \oplus T_{2}\right)$ is isomorphic to $C^{*}(S)$, where $S$ denotes the unilateral shift of multiplicity 1 . Since $S$ is irreducible, we conclude that $C^{*}\left(T_{1} \oplus T_{2}\right)$ does not splits. Hence we have:

COROLlary 2.4. Let $T_{1}$ and $T_{2}$ be isometries with von Neumann-Wold decompositions $U_{i} \oplus S_{i}, i=1,2$. Then $C^{*}\left(T_{1} \oplus T_{2}\right)$ splits if and only if $S_{1}=S_{2}=0$ and $\Lambda\left(U_{1}\right) \cap A\left(U_{2}\right)=\varnothing$. 
Let $T_{1}$ and $T_{2}$ be two compact operators acting on a Hilbert space $\mathscr{H}$. If $\mathscr{H}$ is infinite-dimensional, then it is easy to see that the $C^{*}$-algebra generated by $T_{1} \oplus T_{2}$ and the identity operator on $\mathscr{K} \oplus \mathscr{\mathscr { C }}$ never splits. However, if we consider $C^{*}\left(T_{1} \oplus T_{2}\right)$ in $\mathscr{Y}^{\prime}\left(\mathscr{K} \oplus \mathscr{K}^{\prime}\right)$ (the $C^{*}$-algebra of all compact operators on $\mathscr{H} \oplus \mathscr{K}$ ), then the splitting of $C^{*}\left(T_{1} \oplus T_{2}\right)$ can be characterized as follows:

Corollary 2.5. Let $T_{1}$ and $T_{2}$ be compact operators on $\mathscr{Y}$. Then $C^{*}\left(T_{1} \oplus T_{2}\right)$ (generated as a $C^{*}$-subalgebra of $\mathscr{K}\left(\mathscr{L} \oplus \oplus \mathscr{K}^{*}\right)$ ) splits if and only if every minimal projection in $C^{*}\left(T_{1} \oplus T_{2}\right)$ is of the form $P_{1} \oplus 0$ or $0 \oplus P_{.}$, where $P_{i}$ is a minimal projection in $C^{*}\left(T_{2}\right), i=1,2$.

Proof. $\Leftrightarrow)$. Clear.

$\Leftrightarrow)$. Let. $\mathscr{C}=C^{*}\left(T_{1} \oplus T_{2}\right), \mathscr{O}_{i}=C^{*}\left(T_{i}\right), i=1,2$. Let $\mathscr{l}_{1}$ (resp. . $\left.\mathscr{L}_{2}\right)$ denote the set of minimal projections of $\mathscr{A}$ of the form $P_{1} \oplus 0$ (resp. $0 \oplus P_{2}$ ), where $P_{\imath}$ is a minimal projection in $\mathscr{L}_{i}, i=1,2$. Then by hypothesis,

$\{$ minimal projections in $\mathscr{\mathscr { C }}\}=. / \check{C}_{1} \cup \cdot \mathscr{C}_{2}$.

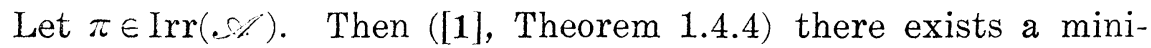
mal projection $P=P_{\pi} \in \mathscr{K}$, a nonzero vector $\xi=\xi_{\pi} \in P(\mathscr{C} \oplus \mathscr{K})$, and a unitary operator $U=U_{n}:[\propto \xi] \rightarrow \mathscr{Y}$ such that

(i ) $\pi(P) \neq 0$,

(ii) $\pi\left(T_{1} \oplus T_{2}\right)=U\left(T_{1} \oplus T_{2}\right) Q U^{*}$, where $Q=$ projection of $\oplus \mathscr{H}$ onto $\mid . \mathscr{A} \xi]$.

We denote this by writing $\pi \sim \mathrm{id}_{P^{\prime}}$. By (2.7), $P$ must be in either or . $\mathscr{H}_{2}$; suppose $P \in \mathscr{H}_{1}$, i.e., $P=R \oplus 0, R$ a minimal projection in $\mathscr{X}_{1}$. Then $\xi=\left(\xi^{\prime}, 0\right), \xi^{\prime}$ a nonzero vector in $R(\mathscr{C})$, and so

$$
[\mathscr{2} \xi]=\left[\cdot \mathscr{R}_{1} \xi^{\prime}\right] \oplus(0) \text {. }
$$

Therefore, there exists a unitary $U^{\prime}:\left[\cdot \mathscr{O}_{1} \xi^{\prime}\right] \rightarrow \mathscr{C}_{*}$ such that $U:(x, 0) \rightarrow U^{\prime} x, x \in\left[\mathscr{\alpha}_{1} \xi^{\prime}\right]$. Thus by (ii),

$$
\left.\pi\left(T_{1} \oplus T_{2}\right)=U^{\prime} T_{1} Q^{\prime}\left(U^{\prime}\right)^{*}, \quad Q^{\prime}=\text { projection of } \mathscr{C}^{\prime} \text { onto [. } \mathscr{L}_{1} \xi^{\prime}\right] .
$$

But by ([1], Proposition 1.4.3), the right side of (2.8) defines an irreducible representation $\sigma$ of $\mathscr{A}$. Thus $\pi=\widetilde{\sigma}$. If $P \in \mathscr{C} / \mathscr{L}_{2}$, the same argument shows that $\tau=\tilde{\tau}$ for some irreducible representation $\tau$ of $\mathscr{L}_{2}$. If $\Sigma_{1}$ and $\Sigma_{2}$ are as defined in Theorem 2.1, we conclude that

$$
\operatorname{Prim}(\mathscr{D})=\Sigma_{1} \cup \Sigma_{2}
$$

We now assert that 


$$
\begin{aligned}
& \Sigma_{1}=\text { hull } \mathscr{C}_{2}=\left\{\operatorname{ker} \pi: \pi \in \operatorname{Irr}(\mathscr{A}), \mathscr{C}_{2} \subseteq \operatorname{ker} \pi\right\}, \\
& \Sigma_{2}=\text { hull } \mathscr{/} \mathscr{l}_{1}=\left\{\operatorname{ker} \pi: \pi \in \operatorname{Irr}(\mathscr{A}), \mathscr{C}_{1} \subseteq \operatorname{ker} \pi\right\} .
\end{aligned}
$$

It is clear from the definition of $\mathscr{C}_{2}$ that $\Sigma_{1} \subseteq$ hull $\mathscr{C}_{2}$. Suppose $\operatorname{ker} \pi \in \operatorname{hull}\left(\mathscr{C}_{2}\right)$. Now $\pi \sim \mathrm{id}_{P}$, with $P \in \mathscr{C}_{1} \cup \mathscr{C}_{2}$. If $P \in \mathscr{C}_{2}$, then $\pi(P)=0$, which by (i) is contrary to the choice of $P$. Thus $P \in \mathscr{A}_{1}$ whence by the previous reasoning, $\pi \in \Sigma_{1}$. This verifies (2.10), and (2.11) follows similarly.

Suppose $\operatorname{ker} \pi \in \Sigma_{1} \cap \Sigma_{2}$. Then $\mathscr{C l}_{1} \cup \mathscr{C}_{2} \subseteq \operatorname{ker} \pi$. But $\pi \sim \mathrm{id}$, for some $P \in \cdot \mathscr{C}_{1} \cup$.// 2 with $\pi(P) \neq 0$, a contradiction. Thus

$$
\Sigma_{1} \cap \Sigma_{2}=\varnothing \text {. }
$$

It follows from (2.9)-(2.12) that $\left\{\Sigma_{1}, \Sigma_{2}\right\}$ disconnects $\operatorname{Prim}(\mathscr{A})$, whence by Theorem 2.1, $C^{*}\left(T_{1} \oplus T_{2}\right)$ splits.

Let $\rho$ be natural map from $\mathscr{B}(\mathscr{H})$ onto the Calkin algebra $\mathscr{B}(\mathscr{H}) / \mathscr{K}(\mathscr{H})$. The following concept is also seen in [12].

Definition. Let $T$ be an element in $\mathscr{B}(\mathscr{H})$. A projection $P$ in $\mathscr{B}(\mathscr{C})$ is fully n-reducing for $T$ if $T P=P T$, rank $(P)<\infty$, and $C^{*}(T) P \cong M_{n}$, the $n \times n$ matrix algebra. A projection $P$ in $\mathscr{B}(\mathscr{H})$ is essentially fully n-reducing for $T$ if $\rho(P) \rho(T)=\rho(T) \rho(P)$, $P$ has infinite rank and nullity, and $\rho\left(C^{*}(T)\right) \rho(P) \cong M_{n}$. We denote the set of all fully (essentially fully) $n$-reducing projections for $T$ by $R_{n}(T)\left(R_{n}^{e}(T)\right)$, and let $R(T)=\bigcup_{n} R_{n}(T), R^{e}(T)=\bigcup_{n} R_{n}^{e}(T)$, where $n$ ranges through all positive integers. Each $P$ in $R^{e}(T)$ (or in $R(T)$ ) induces an irreducible representation, $\pi_{P}$, of $C^{*}(T)$ in a natural way as:

$$
\pi_{P}(A)=\rho(A) \rho(P) \quad\left(\pi_{P}(A)=A P\right) \quad \text { for all } A \text { in } C^{*}(T) .
$$

Definition. Let $T$ and $S$ be elements in $C^{*}$-algebras $\mathscr{A}$ and $\mathscr{B}$ respectively. $T$ is algebraically equivalent to $S$, if there exists a *-isomorphism $\varphi$ of $C^{*}(T)$ onto $C^{*}(S)$ with $\varphi(T)=S$.

Proposition 2.6. Let $T_{i}, i=1,2$, be two operators in $\mathscr{B}(\mathscr{C})$ such that every irreducible representation of $C^{*}\left(T_{i}\right), i=1,2$, has a finite-demensional representation space. $C^{*}\left(T_{1} \oplus T_{2}\right), a C^{*}$-subalgebra of $\mathscr{B}(\mathscr{H} \oplus \mathscr{H})$, splits if and only if the following two conditions hold:

(i) If an operator in $C^{*}\left(T_{1}\right) \oplus C^{*}\left(T_{2}\right)$ is of the form $P_{1} \oplus 0$ or $0 \oplus P_{2}$, where $P_{i}$ is in $R\left(T_{i}\right) \cap C^{*}\left(T_{i}\right), i=1,2$, then it is in $C^{*}\left(T_{1} \oplus T_{2}\right)$.

(ii) If $P_{i} \in R^{e}\left(T_{i}\right), i=1,2$, then $\rho\left(P_{1} T_{1}\right)$ is not algebraically 
equivalent to $\rho\left(P_{2} T_{2}\right)$.

Proof. Let $\mathscr{A}$ be $C^{*}\left(T_{1} \oplus T_{2}\right), \mathscr{A}_{i}$ be $C^{*}\left(T_{i}\right), i=1,2$, and $\Sigma_{i}$ be as in Theorem 2.1, $i=1,2$.

$\left(\Leftrightarrow\right.$ Condition (i) follows from the fact $C^{*}\left(T_{1}\right) \oplus 0$ and $0 \oplus C^{*}\left(T_{2}\right)$ are contained in $\mathscr{A}_{1} \oplus \mathscr{A}_{2}=\mathscr{A}$.

(ii) Let $P_{i}$ be in $R^{e}\left(T_{i}\right), i=1,2$. If there exists a *-isomorphism $\varphi$ of $C^{*}\left(\rho\left(T_{1} P_{1}\right)\right)$ onto $C^{*}\left(\rho\left(T_{2} P_{2}\right)\right)$, with $\varphi\left(\rho\left(T_{1} P_{1}\right)\right)=\rho\left(T_{2} P_{2}\right)$, then the kernels of the two irreducible representations $\tilde{\pi}_{1}, \tilde{\pi}_{2}$ of $\mathscr{A}$ induced by $P_{1}, P_{2}\left(\pi_{i}=\pi_{P_{i}}\right.$ as in (2.13)) are equal. Since ker $\tilde{\pi}_{i}$ is in $\Sigma_{i}$, $i=1,2$, this contradicts the fact that $\Sigma_{1} \cap \Sigma_{2}=\varnothing$ by Theorem 2.1.

$\Leftrightarrow$ Any $\pi$ in $\operatorname{Irr}\left(\mathscr{A}_{1} \oplus \mathscr{A}_{2}\right)$ is of the form $\pi=\tilde{\sigma}_{i}$ for some $\sigma_{i}$ in $\operatorname{Irr}\left(\mathscr{A}_{i}\right)$, and hence is finite-dimensional. Since $\mathscr{A}_{1} \oplus \mathscr{A}_{2}$ is CCR, any two irreducible representations $\pi_{1}, \pi_{2}$ of $\mathscr{A}_{1} \oplus \mathscr{A}_{2}$ are unitarily equivalent if and only if $\operatorname{ker} \pi_{1}=\operatorname{ker} \pi_{2}([8], 4.3 .7)$. $\mathscr{A}$, a $C^{*}$-subalgebra of $\mathscr{A}_{1} \oplus \mathscr{A}_{2}$, is also CCR, and also has the above property. Next, we state a proposition ([8], 11.1.6), and then use the proposition to show that $\mathscr{A}$ splits.

Proposition. Let $\mathscr{B}$ be a $C^{*}$-algebra, and $\mathscr{B}_{1}$ a $C^{*}$-subalgebra of $\mathscr{B}$. If $\mathscr{B}_{1}$ satisfies the following two conditions:

(i) $\left.\pi\right|_{\mathscr{B}_{1}}$ is in $\left.\operatorname{Irr}(\mathscr{B})_{1}\right)$, if $\pi$ is in $\operatorname{Irr}(\mathscr{B})$;

(ii) $\left.\pi\right|_{\mathscr{C}_{1}}$ is not unitarily equivalent to $\left.\pi^{\prime}\right|_{\mathscr{G}_{1}}$, if $\pi$ is not unitarily equivalent to $\pi^{\prime}$ in $\operatorname{Irr}(\mathscr{B})$, then $\mathscr{B}_{1}=\mathscr{B}$.

Let $\pi$ be in $\operatorname{Irr}\left(\mathscr{A}_{1} \oplus \mathscr{A}_{2}\right)$ and of the form $\pi=\tilde{\sigma}_{i}$ for some $\sigma_{i}$ in $\operatorname{Irr}\left(\mathscr{A}_{i}\right)$. So $\pi\left(T_{1} \oplus T_{2}\right)=\widetilde{\sigma}_{i}\left(T_{1} \oplus T_{2}\right)=\sigma_{i}\left(T_{i}\right)$, whence $\pi(\mathscr{A})=$ $\sigma_{i}\left(\mathscr{L}_{i}\right)$ on $\mathscr{H}_{\pi}$, and $\left.\pi\right|_{\infty}$ is irreducible. Let $\pi$ be an $n$-dimensional irreducible representation of $C^{*}(T)$ for some $T$ in $\mathscr{B}(\mathscr{H})$. Theorem 1.1 in [12] implies that either (a) $\exists P \in C^{*}(T) \cap R(T)$ such that $\pi(P)=1$ and the restriction of $\pi$ to $C^{*}(T) P$ is a *-isomorphism of $C^{*}(T) P$ onto $M_{n}$, or (b) $\exists P \in R^{e}(T)$ and a *-isomorphism $\varphi$ of $\rho\left(C^{*}(T)\right) \rho(P)$ onto $M_{n}$ such that $\pi(A)=\varphi(\rho(A) \rho(P))\left(A \in C^{*}(T)\right)$.

Suppose $\pi_{1}, \pi_{2}$ are two unitarily inequivalent elements in $\operatorname{Irr}\left(\mathscr{A}_{1} \oplus \mathscr{A}_{2}\right)$, and $\pi_{i}=\tilde{\sigma}_{i}$ for $\sigma_{i}$ in $\operatorname{Irr}\left(\mathscr{A}_{j(i)}\right), i=1,2$.

Case 1. $j(1)=j(2)$. We note $\pi_{i}\left(T_{1} \oplus T_{2}\right)=\widetilde{\sigma}_{i}\left(T_{1} \oplus T_{2}\right)=\sigma_{i}\left(T_{j(i)}\right)$, $i=1,2$, and unitary equivalence between $\left.\pi_{1}\right|_{\mathscr{N}}$ and $\left.\pi_{2}\right|_{\infty}$ implies that there exists a ${ }^{*}$-isomorphism $\varphi$ of $\pi_{1}(\mathscr{A})$ onto $\pi_{2}(\mathscr{A})$ with $\varphi\left(\sigma_{1}\left(T_{j(i)}\right)=\right.$ $\sigma\left(\pi_{1}\left(T_{1} \oplus T_{2}\right)\right)=\pi_{2}\left(T_{1} \oplus T_{2}\right)=\sigma_{2}\left(T_{j(2)}\right)$. This $\varphi$ induces a unitary equivalence between $\sigma_{1}\left(\mathscr{A}_{j(1)}\right)$ and $\sigma_{2}\left(\mathscr{A}_{j(2)}\right)$. If $A_{1} \oplus A_{2}$ are in $\mathscr{A}_{1} \oplus \mathscr{A}_{2}, \varphi\left(\tilde{\sigma}_{1}\left(A_{1} \oplus A_{2}\right)\right)=\varphi\left(\sigma_{1}\left(A_{j(1)}\right)\right)=\sigma_{2}\left(A_{j(1)}\right)=\sigma_{2}\left(A_{j(2)}\right)=\tilde{\sigma}_{2}\left(A_{1} \oplus A_{2}\right)$. The second equality in this equation is due to a property of $\varphi$, which is illustrated in the following commutative diagram: 


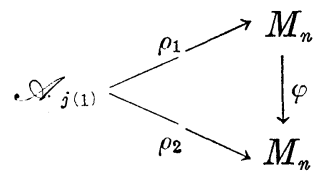

It follows that $\pi_{1}$ and $\pi_{2}$ are unitarily equivalent on $\mathscr{A}_{1} \oplus \mathscr{A}_{2}$, which is a contradiction. Therefore $\left.\pi_{1}\right|_{\mathscr{}}$ is not unitarily equivalent to $\left.\pi_{2}\right|_{\mathscr{A}}$.

Case 2. $j(1) \neq j(2)$. Let $j(1)=1, j(2)=2$. If $\sigma_{1}$ is of form (a) relative to $P$ in $R\left(T_{1}\right) \cap \mathscr{A}_{1}$ with $P \oplus 0$ in $\mathscr{A}$, then $\pi_{1}(P \oplus 0)=$ $\widetilde{\sigma}_{1}(P \oplus 0)=\pi_{1}(P)=I$ and $\pi_{2}(P \oplus 0)=\widetilde{\sigma}_{2}(P \oplus 0)=\sigma_{2}(0)=0$. It follows that $\left.\pi_{1}\right|_{\circlearrowleft \text { s }}$ is not unitarily equivalent to $\left.\pi_{2}\right|_{\mathscr{}}$. Similary $\left.\pi_{1}\right|_{\mathscr{}}$ is not unitarily equivalent to $\left.\pi_{2}\right|_{\mathscr{\sim}}$ if $\pi_{2}$ is of form (a). Suppose both $\pi_{1}$ and $\pi_{2}$ are of form (b), i.e., $\exists P_{i} \in R^{e}\left(T_{i}\right)$ and a ${ }^{*}$-isomorphism $\varphi_{i}$ of $\rho\left(\mathscr{A}_{i}\right) \rho\left(P_{i}\right)$ onto $M_{n_{i}}$ such that $\sigma_{i}(A)=\varphi_{i}\left(\rho(A) \rho\left(P_{i}\right)\right),\left(A \in \mathscr{A}_{i}\right), i=$ 1,2. We note that

$$
\begin{aligned}
& \pi_{1}\left(T_{1} \oplus T_{2}\right)=\widetilde{\sigma}_{1}\left(T_{1} \oplus T_{2}\right)=\widetilde{\sigma}_{1}\left(T_{1}\right)=\varphi_{1}\left(\rho\left(T_{1}\right) \rho\left(P_{1}\right)\right) \\
& \pi_{2}\left(T_{1} \oplus T_{2}\right)=\widetilde{\sigma}_{2}\left(T_{1} \oplus T_{2}\right)=\sigma_{2}\left(T_{2}\right)=\varphi_{2}\left(\rho\left(T_{2}\right) \rho\left(P_{2}\right)\right) .
\end{aligned}
$$

Since $\rho\left(T_{1}\right) \rho\left(P_{1}\right)$ and $\rho\left(T_{2}\right) \rho\left(P_{2}\right)$ are not algebraically equivalent, there exists no ${ }^{*}$-isomorphism of $\varphi_{1}\left(\rho\left(\mathscr{A} \mathscr{A}_{1}\right) \rho\left(P_{1}\right)\right)$ onto $\varphi_{2}\left(\rho\left(\mathscr{A}_{2}\right) \rho\left(P_{2}\right)\right)$, which maps $\varphi_{1}\left(\rho\left(T_{1}\right) \rho\left(P_{1}\right)\right)$ to $\varphi_{2}\left(\rho\left(T_{2}\right) \rho\left(P_{2}\right)\right)$. This implies that there exists no ${ }^{*}$-isomorphism of $\pi_{1}(\mathscr{A})$ onto $\pi_{2}(\mathscr{A})$ which maps $\pi_{1}\left(T_{1} \oplus T_{2}\right)$ to $\pi_{2}\left(T_{1} \oplus T_{2}\right)$. Hence $\left.\pi_{1}\right|_{\mathscr{\leftrightarrow}}$ is not unitarily equivalent to $\left.\pi_{2}\right|_{\mathscr{\leftrightarrow}}$.

In the following we use a Stone-Weierstrass theorem for $C^{*}$-algebras to obtain a significant improvement of Theorem 2.1 in an important special case.

Recall that a subset $\mathscr{B}$ containing the identity of a unital $C^{*}$-algebra $\mathscr{A}$ separates the pure states of $\mathscr{C}$ if to each pair $\rho_{1}$ and $\rho_{2}$ of distinct pure states of $\mathscr{A}$, there corresponds a $B \in \mathscr{B}$ such that $\rho_{1}(B) \neq \rho_{2}(B)$.

We fix a unital $C^{*}$-algebra $\mathscr{A}$ and elements $T_{1}, T_{2}$ of $\mathscr{A} . \Sigma_{1}$ and $\Sigma_{2}$ are defined relative to $C^{*}\left(T_{1} \oplus T_{2}\right)$ as in Theorem 2.1.

LEMma 2.7. If $\Sigma_{1} \cap \Sigma_{2}=\varnothing$, then $C^{*}\left(T_{1} \oplus T_{2}\right)$ separates the pure states of $C^{*}\left(T_{1}\right) \oplus C^{*}\left(T_{2}\right)$.

Proof. Let $\mathscr{A}=C^{*}\left(T_{1}\right) \oplus C^{*}\left(T_{2}\right), \mathscr{P}=C^{*}\left(T_{1} \oplus T_{2}\right), \mathscr{A}_{i}=C^{*}\left(T_{i}\right)$, $i=1,2$.

Suppose $\rho_{1}$ and $\rho_{2}$ are pure states of $\mathscr{A}$ such that $\left.\rho_{1}\right|_{\mathscr{C}}=\left.\rho_{2}\right|_{\mathscr{G}}$. For $i=1,2$, there is an irreducible representation $\pi_{i}$ of $\mathscr{A}$ and 
a unit vector $\xi_{i} \in \mathscr{H}_{\pi_{i}}$ for which $\rho_{i}(\cdot)=\left(\pi_{i}(\cdot) \xi_{i}, \xi_{i}\right)$. Now $\pi_{i}$ is of the form $\tilde{\sigma}$ for $\sigma \in \operatorname{Irr} \mathscr{A}_{1} \cup \operatorname{Irr} \mathscr{A}_{2}$. If $\sigma \in \operatorname{Irr} \mathscr{A}_{1}$, then

$$
\rho_{i}(A \oplus B)=\left(\sigma(A) \xi_{i}, \xi_{i}\right), \quad \forall A \oplus B \in \mathscr{A},
$$

and so $\rho_{i}=\tilde{f}$ for some pure state $f$ on $\mathscr{A}_{1}$. Similarly, $\rho_{i}=\widetilde{g}$ for some pure state $g$ on $\mathscr{A}_{2}$ if $\sigma \in \operatorname{Irr} \mathscr{A}_{2}$.

Suppose $\rho_{i}=\tilde{f}_{i}, f_{i}$ a pure state on $\mathscr{A}_{i}, i=1,2$. We denote by $\mathscr{P}$ the set of all polynomials in two noncommutating variables and for $p \in \mathscr{P}$, we set $p\left(T_{i}\right)=p\left(T_{i}, T_{i}^{*}\right), i=1,2$. Since $\left.\rho_{1}\right|_{\mathscr{B}}=\left.\rho_{2}\right|_{\mathscr{B}}$, it. follows that

$$
f_{1}\left(p\left(T_{1}\right)\right)=f_{2}\left(p\left(T_{2}\right)\right), \quad \forall p \in \mathscr{P} .
$$

Let $\mathscr{C}_{i}=$ GNS Hilbert space corresponding to $f_{i}$, and set $\operatorname{ker} f_{i}=$ $\left\{A \in \mathscr{A}_{i}: f_{i}\left(A^{*} A\right)=0\right\}$. Define the mapping $U: \mathscr{P}\left(T_{1}\right) / \operatorname{ker} f_{1} \rightarrow \mathscr{P}\left(T_{2}\right) /$ $\operatorname{ker} f_{2}$ by $U: p\left(T_{1}\right)+\operatorname{ker} f_{1} \rightarrow p\left(T_{2}\right)+\operatorname{ker} f_{2}, p \in \mathscr{P}$. Then by (1),

$$
\begin{aligned}
\left\|p\left(T_{1}\right)+\operatorname{ker} f_{1}\right\|_{\mathscr{C}_{1}}^{2} & =f_{1}\left(p\left(T_{1}\right)^{*} p\left(T_{1}\right)\right) \\
& =f_{2}\left(p\left(T_{2}\right)^{*} p\left(T_{2}\right)\right) \\
& =\left\|p\left(T_{2}\right)+\operatorname{ker} f_{2}\right\|_{\mathscr{C}_{2}}^{2}, \quad \forall p \in \mathscr{P},
\end{aligned}
$$

and so $U$ extends to a unitary transformation of $\mathscr{H}_{1}$ onto $\mathscr{H}_{2}$. Also, if $p, q \in \mathscr{P}$ and $\pi_{f_{i}}$ is the GNS representation corresponding to $f_{i}$, then

$$
\begin{aligned}
\pi_{f_{2}}\left(p\left(T_{2}\right)\right) U\left(q\left(T_{1}\right)+\operatorname{ker} f_{1}\right) & =\pi_{f_{2}}\left(p\left(T_{2}\right)\right)\left(q\left(T_{2}\right)+\operatorname{ker} f_{2}\right) \\
& =p\left(T_{2}\right) q\left(T_{2}\right)+\operatorname{ker} f_{2} \\
& =U\left(p\left(T_{1}\right) q\left(T_{1}\right)+\operatorname{ker} f_{1}\right) \\
& =U \pi_{f_{1}}\left(p\left(T_{1}\right)\right)\left(q\left(T_{1}\right)+\operatorname{ker} f_{1}\right) .
\end{aligned}
$$

Since $p$ and $q$ are arbitrary, it follows that $\left.\tilde{\pi}_{f_{1}}\right|_{\mathscr{\theta}}$ is unitarily equivalent to $\left.\tilde{\pi}_{f_{2}}\right|_{\mathscr{\alpha}}$, and so $\operatorname{ker}\left(\left.\tilde{\pi}_{f_{1}}\right|_{\mathscr{B}}\right)=\operatorname{ker}\left(\left.\tilde{\pi}_{f_{2}}\right|_{\mathscr{O}}\right) \in \Sigma_{1} \cap \Sigma_{2}$, contrary to assumption.

We conclude that either

(a) $\rho_{i}=\widetilde{\sigma}_{i}, \sigma_{i}$ a pure state on $\mathscr{A}_{1}, i=1,2$,

or

(b) $\rho_{i}=\tilde{\sigma}_{i}, \sigma_{i}$ a pure state on $\mathscr{A}_{2}, i=1,2$.

Suppose (a) holds. Let $p, q \in \mathscr{P}$. We have

$$
\begin{aligned}
& \rho_{1}\left(p\left(T_{1}\right) \oplus q\left(T_{2}\right)\right)=\sigma_{1}\left(p\left(T_{1}\right)\right), \\
& \rho_{2}\left(p\left(T_{1}\right) \oplus q\left(T_{2}\right)\right)=\sigma_{2}\left(p\left(T_{1}\right)\right) .
\end{aligned}
$$

Now $p\left(T_{1}\right) \oplus p\left(T_{2}\right) \in \mathscr{B}$, and so since $\left.\rho_{1}\right|_{\mathscr{O}}=\left.\rho_{2}\right|_{\mathscr{O}}$,

$$
\sigma_{1}\left(p\left(T_{1}\right)\right)=\sigma_{2}\left(p\left(T_{1}\right)\right)
$$


Thus by (2.14), (2.15), (2.16), and the arbitrariness of $p$ and $q, \rho_{1}=\rho_{2}$. For case (b), argue similarly.

THEOREM 2.8. Suppose $C^{*}\left(T_{1} \oplus T_{2}\right)$ is strongly amenable (consult ([11], definition, p. 70). Then $C^{*}\left(T_{1} \oplus T_{2}\right)$ splits if and only if $\Sigma_{1} \cap \Sigma_{2}=\varnothing$.

Proof. We need only verify the "if" part. By Lemma 2.7, $C^{*}\left(T_{1} \oplus T_{2}\right)$ separates the pure states of $C^{*}\left(T_{1}\right) \oplus C^{*}\left(T_{2}\right)$. Thus by Proposition 3.3 in [3], $C^{*}\left(T_{1} \oplus T_{2}\right)=C^{*}\left(T_{2}\right) \oplus C^{*}\left(T_{1}\right)$.

CoROLlary 2.9. Suppose $T_{1}$ and $T_{2}$ are GCR elements (i.e., $C^{*}\left(T_{i}\right)$ is a GCR algebra, $\left.i=1,2\right)$. Then $C^{*}\left(T_{1} \oplus T_{2}\right)$ splits if and only if $\Sigma_{1} \cap \Sigma_{2}=\varnothing$.

Proof. Since all GCR algebras are strongly amenable ([11], Theorem 7.9 , p. 78), this corollary is evident from the above theorem.

REMARK 2.10. Theorem 2.8 (and hence Corollary 2.9) also holds in the nonunital case. One need only check that there can exist no nonzero pure state of $C^{*}\left(T_{1}\right) \oplus C^{*}\left(T_{2}\right)$ which vanishes on $C^{*}\left(T_{1} \oplus T_{2}\right)$, and this follows from the fact that each pure state of $C^{*}\left(T_{1}\right) \oplus C^{*}\left(T_{2}\right)$ is "evaluation at coordinates" of a pure state of either $C^{*}\left(T_{1}\right)$ or $C^{*}\left(T_{2}\right)$ (see the beginning of the proof of Lemma 2.7).

3. The splitting of $W^{*}\left(T_{1} \oplus T_{2}\right)$. In this section necessary and sufficient conditions for the splitting of $W^{*}\left(T_{1} \oplus T_{2}\right)$ are given, where $T_{i} \in \mathscr{B}\left(\mathscr{C}_{i}\right)$ for Hilbert spaces $\mathscr{K}_{i}, i=1,2$.

We begin by considering a slightly more general problem. Let

$$
\begin{aligned}
& \mathscr{S}=\left\{T_{1} \oplus 0,0 \oplus T_{2}, T_{1}^{*} \oplus 0,0 \oplus T_{2}^{*}\right\}, \\
& \mathscr{F}=\left\{T_{1} \oplus T_{2}, T_{1}^{*} \oplus T_{2}^{*}\right\}, \\
& \mathscr{Y}=\mathscr{S} \cup\{I \oplus 0\} .
\end{aligned}
$$

We are interested in deriving conditions under which the $W^{*}$-algebras generated by $\mathscr{S}, \mathscr{F}$, and $\mathscr{V} \cdot$ coincide. By the double commutant theorem, it suffices to consider $\mathscr{S}^{\prime \prime}, \mathscr{F} "$, and $\mathscr{V}^{\prime \prime}$ (' denotes commutant), and we easily see that $\mathscr{F}^{\prime \prime} \subseteq \mathscr{S}^{\prime \prime} \cong \mathscr{V}^{\prime \prime}$.

Let $S=\left(\begin{array}{ll}S_{11} & S_{12} \\ S_{21} & S_{22}\end{array}\right)$ be in $\mathscr{F}^{\prime}$, with $S^{*}=S$, i.e., $S_{i i}^{*}=S_{i i}, i=1,2$, and $S_{12}=S_{21}^{*}$. From

$$
S\left(\begin{array}{cc}
T_{1} & 0 \\
0 & T_{2}
\end{array}\right)=\left(\begin{array}{cc}
T_{1} & 0 \\
0 & T_{2}
\end{array}\right) S
$$


it follows that

$$
\left(\begin{array}{llll}
S_{11} & T_{1} & S_{12} & T_{2} \\
S_{22} & T_{1} & S_{22} & T_{2}
\end{array}\right)=\left(\begin{array}{llll}
T_{1} & S_{11} & T_{1} & S_{12} \\
T_{2} & S_{21} & T_{2} & S_{22}
\end{array}\right)
$$

Thus we have

$$
\begin{aligned}
& S_{i i} T_{i}=T_{i} S_{i i}, i=1,2 \\
& S_{12} T_{2}=T_{1} S_{12}, \\
& S_{12}^{*} T_{1}=T_{2} S_{12}^{*} .
\end{aligned}
$$

Similarly from

$(3.1)^{*}$

$$
\begin{aligned}
S\left(\begin{array}{cc}
T_{1}^{*} & 0 \\
0 & T_{2}^{*}
\end{array}\right) & =\left(\begin{array}{ll}
T_{1}^{*} & 0 \\
0 & T_{2}^{*}
\end{array}\right) S, \text { we obtain } \\
S_{i i} T_{i}^{*} & =T_{i}^{*} S_{i i}, i=1,2 \\
S_{12} T_{2}^{*} & =T_{1}^{*} S_{12} \\
S_{12}^{*} T_{1}^{*} & =T_{2}^{*} S_{12}^{*} .
\end{aligned}
$$

Since $(3.1)^{*}$ is just the "adjoint" version of (3.1), we have the following lemma:

LEMMA 3.1. Let $S^{*}=S=\left(\begin{array}{ll}S_{11} & S_{12} \\ S_{21} & S_{22}\end{array}\right)$ be in $\mathscr{B}\left(\mathscr{C}_{1} \oplus \mathscr{C _ { 2 }}\right)$. Then $S \in \mathscr{F}^{\prime}$ if and only if

$$
\begin{aligned}
& S_{i i} T_{i}=T_{i} S_{i i}, i=1,2, \\
& S_{12} T_{2}=T_{1} S_{12} \\
& S_{12}^{*} T_{1}=T_{2} S_{12}^{*} .
\end{aligned}
$$

Now suppose $S \in \mathscr{S}^{\prime}$ and $S=S^{*}$. From

$$
S\left(\begin{array}{ll}
T_{1} & 0 \\
0 & 0
\end{array}\right)=\left(\begin{array}{ll}
T_{1} & 0 \\
0 & 0
\end{array}\right) S \text { and } S\left(\begin{array}{ll}
0 & 0 \\
0 & T_{2}
\end{array}\right)=\left(\begin{array}{cc}
0 & 0 \\
0 & T_{2}
\end{array}\right) S
$$

we get

$$
\begin{aligned}
& T_{i} S_{i i}=S_{i i} T_{i}, i=1,2, \\
& S_{12}^{*} T_{1}=T_{1} S_{12}=S_{12} T_{2}=T_{2} S_{12}^{*}=0 .
\end{aligned}
$$

Similarly from

$$
S\left(\begin{array}{ll}
T_{1}^{*} & 0 \\
0 & 0
\end{array}\right)=\left(\begin{array}{ll}
T_{1}^{*} & 0 \\
0 & 0
\end{array}\right) S \text { and } S\left(\begin{array}{lr}
0 & 0 \\
0 & T_{2}^{*}
\end{array}\right)=\left(\begin{array}{rr}
0 & 0 \\
0 & T_{2}^{*}
\end{array}\right) S
$$

we get 


$$
\begin{aligned}
& T_{i}^{*} S_{i i}=S_{i i} T_{i}^{*}, i=1,2, \\
& S_{12}^{*} T_{1}^{*}=T_{1}^{*} S_{12}=S_{12} T_{2}^{*}=T_{2}^{*} S_{12}^{*}=0 .
\end{aligned}
$$

Therefore we have

LEMMA 3.2. Let $S^{*}=S=\left(\begin{array}{ll}S_{11} & S_{12} \\ S_{21} & S_{22}\end{array}\right)$ be in $\mathscr{B}\left(\mathscr{C}_{1} \oplus \mathscr{Y}_{2}\right)$. Then $S \in \mathscr{S}^{\prime}$ if and only if

$$
\begin{aligned}
& S_{i i} T_{i}=T_{i} S_{i i}, i=1,2, \\
& S_{12} T_{2}=T_{1} S_{12}=S_{12}^{*} T_{1}=T_{2} S_{12}^{*}=0 .
\end{aligned}
$$

Finally if $S \in \mathscr{V}^{\prime}$ if follows from

$$
S\left(\begin{array}{ll}
I & 0 \\
0 & 0
\end{array}\right)=\left(\begin{array}{ll}
I & 0 \\
0 & 0
\end{array}\right) S \text { that }\left(\begin{array}{rr}
S_{11} & S_{12} \\
0 & 0
\end{array}\right)=\left(\begin{array}{ll}
S_{11} & 0 \\
S_{12}^{*} & 0
\end{array}\right), \quad \text { where } S_{12}=0 .
$$

The following theorem is an immediate consequence of Lemmas 3.1 and 3.2.

THEOREM 3.3. (1). $\mathscr{F}^{\prime \prime}=\mathscr{S}^{\prime \prime}$ if and only if for any bounded linear operator $S$ from $\mathscr{C}_{2}$ into $\mathscr{C}_{1}$ we have $S T_{2}=S^{*} T_{1}=0$ whenever $S T_{2}=T_{1} S$ and $S^{*} T_{1}=T_{2} S^{*}$.

(2) $\mathscr{S}^{\prime \prime}=\mathscr{V}^{\prime \prime}$ if and only if for any bounded linear operator $S$ from $\mathscr{C}_{2}$ into $\mathscr{C}_{1}$ we have $S=0$ whenever $S T_{2}=T_{1} S=S^{*} T_{1}=$ $T_{2} S^{*}=0$.

(3) $\mathscr{F}^{\prime \prime}=\mathscr{V}^{\prime \prime}$ if and only if for any bounded linear operator $S$ from $\mathscr{C}_{2}$ into $\mathscr{C}_{1}$ we have $S=0$ whenever $S T_{2}=T_{1} S$ and $S^{*} T_{1}=T_{2} S^{*}$.

Let $\mathscr{N}$ be a $W^{*}$-algebra, $\mathscr{N}_{*}$ its predual, and let $\operatorname{Rep}_{\sigma}(\mathscr{N})$ denote the family of all $\sigma\left(\mathscr{N}, \mathscr{N}_{*}\right)$-continuous representations of $\mathscr{N}$. Each point of the positive part of the unit ball of $\mathscr{N}_{*}$ gives rise to an element of $\operatorname{Rep}_{o}(\mathscr{N})$ via the Gelfand-Naimark-Segal construction, and therefore $\operatorname{Rep}_{\sigma}(\mathscr{N})$ separates points in $\mathscr{N}$.

Now, let $T_{i} \in \mathscr{B}\left(\mathscr{H}_{i}\right), i=1,2$, and set $\mathscr{N}=W^{*}\left(T_{1} \oplus T_{2}\right)$, $\mathscr{N}_{i}=W^{*}\left(T_{i}\right), i=1,2$. For $\pi \in \operatorname{Rep}_{o}\left(\mathscr{N}_{i}\right)$, defined as in $\S 2$, $\tilde{\pi}\left(T_{1} \oplus T_{2}\right)=\pi\left(T_{i}\right)$. Then $\tilde{\pi} \in \operatorname{Rep}_{\sigma}(\mathscr{N})$. There hence exists a central projection $P=P_{\tilde{\pi}} \in \mathscr{N}$ such that ker $\tilde{\pi}=\mathscr{N} P$. Let supp $\tilde{\pi}=I-P$, and let

$$
\Pi_{i}=\left\{\operatorname{supp} \tilde{\pi}: \pi \in \operatorname{Rep}_{\sigma}\left(\mathscr{N}_{i}\right)\right\}, \quad i=1,2 .
$$

Suppose that

$$
\left.\Pi_{i} \perp \Pi_{2} \text { (i.e., } S_{1} S_{2}=0, \quad S_{i} \in \Pi_{i}, \quad i=1,2\right),
$$


$\sup \left(\Pi_{1} \cup \Pi_{2}\right) \equiv \sup \left\{P: P \in \Pi_{1} \cup \Pi_{2}\right\}=I=$ identity on $\mathscr{H}_{1} \oplus \mathscr{H}_{2}$. Let $P_{i}=\sup \left\{P: P \in \Pi_{i}\right\}, i=1,2 . \quad P_{i}$ is a central projection in $\mathscr{N}$, and by $\left(^{*}\right), P_{1} \perp P_{2}, P_{1}+P_{2}=I$. Let $Q=P_{1}$, so that $I-Q=P_{2}$. Let $Q=Q_{1} \oplus Q_{2}$.

Since $Q=P_{1}, P_{1} \perp P_{2}$, and $\pi\left(Q_{2}\right)=\tilde{\pi}(Q)=\tilde{\pi}\left(P_{1}\right)=0$ for all $\pi$ in $\operatorname{Rep}_{\sigma}\left(\mathscr{N}_{2}\right)$, we conclude that $Q_{2}=0$. Similarly, for all $\pi$ in $\operatorname{Rep}_{o}\left(\mathscr{N}_{1},\right)$ we have

$$
\begin{aligned}
\pi\left(I_{1}-Q_{1}\right) & =\pi\left(I_{1}\right)-\pi\left(Q_{1}\right) \\
& =I-\tilde{\pi}(Q) \\
& =I-\tilde{\pi}\left(P_{1}\right) \\
& =I-I=0 .
\end{aligned}
$$

Hence $I_{1}=Q_{1}$. Therefore $Q=I_{1} \oplus 0$, and $W^{*}\left(T_{1} \oplus T_{2}\right)$ splits.

From the preceding discussion and Theorem 3.3, we may hence deduce the following result, which gives spatial and space-free criteria for the splitting of $W^{*}\left(T_{1} \oplus T_{2}\right)$.

Theorem 3.4. Let $T_{i} \in \mathscr{B}\left(\mathscr{H}_{i}\right), i=1,2$. The following are equivalent:

(a) $W^{*}\left(T_{1} \oplus T_{2}\right)$ splits.

(b) $\Pi_{1} \perp \Pi_{2}$ and $\sup \left(\Pi_{1} \cup \Pi_{2}\right)=I$.

(c) For any bounded linear operator $S$ from $\mathscr{H}_{2}$ into $\mathscr{H}_{1}$, we have $S=0$ whenever $S T_{2}=T_{1} S$ and $S^{*} T_{1}=T_{2} S^{*}$.

Furthermore, $W^{*}\left(T_{1} \oplus T_{2}\right)$ splits if either $W^{*}\left(\operatorname{Re} T_{1} \oplus \operatorname{Re} T_{2}\right)$ or $W^{*}\left(\operatorname{Im} T_{1} \oplus \operatorname{Im} T_{2}\right)$ splits.

Proof. $\quad(a) \Leftrightarrow(b)$. This follows immediately from the discussion following Theorem 3.3.

(a) $\Leftrightarrow(c)$. Notice first that by the double commutant theorem, $W^{*}\left(T_{1} \oplus T_{2}\right)$ splits precisely when $\mathscr{F}^{\prime \prime}=\mathscr{V}^{\prime \prime}$. Now apply Theorem $3.3(3)$.

Suppose $W^{*}\left(\operatorname{Re} T_{1} \oplus \operatorname{Re} T_{2}\right)$ splits. Let $S$ be a bounded linear operator from $H_{2}$ into $H_{1}$ such that $S T_{2}=T_{1} S$ and $S^{*} T_{1}=T_{2} S^{*}$. Then $T_{1} S=S T_{2}^{*}$, so

$$
\left(\operatorname{Re} T_{1}\right) S=\frac{T_{1}+T_{1}^{*}}{2} S=S \frac{T_{2}+T_{2}^{*}}{2}=S\left(\operatorname{Re} T_{2}\right) .
$$

Thus from Theorem 3.3 (3) and the fact that $W^{*}\left(\operatorname{Re} T_{1} \oplus \operatorname{Re} T_{2}\right)$ splits, we conclude that $S=0$. This verifies (c), and so $W^{*}\left(T_{1} \oplus T_{2}\right)$ splits. Argue similarly if $W^{*}\left(\operatorname{Im} T_{1} \oplus \operatorname{Im} T_{2}\right)$ splits.

REMARK 3.5. We now show by example that $W^{*}\left(T_{1} \oplus T_{2}\right)$ can split with neither $W^{*}\left(\operatorname{Re} T_{1} \oplus \operatorname{Re} T_{2}\right)$ nor $W^{*}\left(\operatorname{Im} T_{1} \oplus \operatorname{Im} T_{2}\right)$ split- 
ting.

Let $\alpha_{n}=1 / n, \beta_{n}=1 / n+i, n=1,2,3, \cdots$. Let $T_{1}\left(\operatorname{resp} . T_{2}\right)$ be the diagonal operator with diagonal $\left\{\alpha_{1}, \beta_{2}, \alpha_{3}, \beta_{4}, \cdots\right\}$ (resp. $\left\{\beta_{1}\right.$, $\left.\alpha_{2}, \beta_{3}, \alpha_{4}, \cdots\right\}$ ), acting on the separable Hilbert space $H$. We have

$$
\begin{aligned}
& \Lambda\left(T_{1}\right)=\{0, i\} \cup\left\{\alpha_{1}, \beta_{2}, \alpha_{3}, \cdots\right\}, \\
& \Lambda\left(T_{2}\right)=\{0, i\} \cup\left\{\beta_{1}, \alpha_{2}, \beta_{2}, \cdots\right\} .
\end{aligned}
$$

If $A$ and $B$ are normal operators, it follows from Theorem 3.4 (b) or ([9], Theorem 4.71) that $W^{*}(A \oplus B)$ splits if and only if a scalar spectral measure of $A$ is orthogonal to a scalar spectral measure of $B$. Let $E_{k}$ denote the projection-valued spectral measure of $T_{k}, k=1,2$. If $\left\{\chi_{n}\right\}$ is a countable dense subset of the unit ball of $\mathscr{C}$, then

$$
\mu_{k}(\cdot)=\sum_{n=1}^{\infty} 2^{-n}\left\|E_{k}(\cdot) \chi_{n}\right\|^{2}
$$

is a scalar spectral measure for $T_{k}, k=1,2$. Since 0 and $i$ are not eigenvalues of $T_{k}, k=1,2$, it follows by ([14], Theorem 12.29) that $\mu_{k}(\{0, i\})=0, k=1,2$. Since $\mu_{k}$ is supported on $\Lambda\left(T_{k}\right), k=1,2$, we conclude that $\mu_{1}$ and $\mu_{2}$ are orthogonal, and so $W^{*}\left(T_{1} \oplus T_{2}\right)$ splits. But one easily checks that $\Lambda\left(\operatorname{Re} T_{1}\right)=\Lambda\left(\operatorname{Re} T_{2}\right), \Lambda\left(\operatorname{Im} T_{1}\right)=\Lambda\left(\operatorname{Im} T_{2}\right)$ and therefore neither $W^{*}\left(\operatorname{Re} T_{1} \oplus \operatorname{Re} T_{2}\right)$ nor $W^{*}\left(\operatorname{Im} T_{1} \oplus \operatorname{Im} T_{2}\right)$ splits. This also provides an example of operators $T_{1}$ and $T_{2}$ such that $W^{*}\left(T_{1} \oplus T_{2}\right)$ splits, but $C^{*}\left(T_{1} \oplus T_{2}\right)$ does not.

\section{REFERENCES}

1. W. B. Arveson, An Invitation to $C^{*}$-Algebras, Springer-Verlag, Berlin-HeidelbergNew York, 1976.

2. L. Brickman and P.A. Fillmore, The invariant subspace lattice of a linear transformation, Canad. J. Math., 19 (1967), 810-822.

3. J. Bunce, Characterizations of amenable and strongly amenable $C^{*}$-algebras, Pacific

J. Math., 43 (1972), 563-572.

4. L. A. Coburn, The $C^{*}$-algebra generated by an isometry, Bull Amer. Math. Soc., 73 (1967), 722-726.

5. J. B. Conway, The direct sum of normal operators, Indiana U. Math. J., 26 (1977), 277-289.

6. J.B. Conway and P.Y. Wu, The splitting of $A\left(T_{1} \oplus T_{2}\right)$ and related questions, Indiana U. Math, J., 26 (1977), 41-56.

7. T. Crimmins and P. Rosenthal, On the decomposition of invariant subspaces, Bull. Amer. Math. Soc., 73 (1967), 97-99.

8. J. Dixmier, Les $C^{*}$-algèbres et leurs représentations, Gauthiervillars, Paris, 1969.

9. R. G. Douglas, Banach Algebra Techniques in Operator Theory, Academic Press, New York, 1972.

10. G. Elliott and D. Olesen, A simple proof of the Dauns-Hofmann theorem, Math. Scand., 34 (1974), 231-234.

11. B.E. Johnson, Cohomology in Banach algebras, Memoirs Amer. Math. Soc., no. 127, 1972. 
12. C. Pearcy and N. Salinas, Finite-dimensional representations of $C^{*}$-algebras and reducing matricial spectra of an operator, Rev. Roum. Math. Pures et appl., Vol. XX, No. 5, 567-598, Bucarest, 1975.

13. G.K. Pedersen, Applications of weak* semicontinuity in $C^{*}$-algebra theory, Duke Math. J., 39 (1972), 431-450.

14. W. Rudin, Functional Analysis, McGraw-Hill, New York, 1973.

15. J. Wermer, Banach Algebras and Several Complex Variables, Springer-Verlag, Berlin-Heidelberg-New York, 1976.

Received August 9, 1978 and in revised form January 3, 1979.

OAKLAND UNIVERSITY

ROCHESTER, MI 48063 



\section{PACIFIC JOURNAL OF MATHEMATICS}

\section{EDITORS}

DONALD BABBITT (Managing Editor)

University of California

Los Angeles, California 90024

Hugo RossI

University of Utah

Salt Lake City, UT 84112

C. C. MOORE and ANDrew OGG

University of California

Berkeley, CA 94720
J. DUgundjI

Department of Mathematics University of Southern California Los Angeles, California 90007

R. FinN aNd J. Milgram Stanford University Stanford, California 94305

\section{ASSOCIATE EDITORS}

E. F. BECKENBACH

B. H. NeUMANN

F. WOLF

K. YosHIDA

\section{SUPPORTING INSTITUTIONS}

UNIVERSITY OF BRITISH COLUMBIA CALIFORNIA INSTITUTE OF TECHNOLOGY UNIVERSITY OF CALIFORNIA MONTANA STATE UNIVERSITY UNIVERSITY OF NEVADA, RENO NEW MEXICO STATE UNIVERSITY OREGON STATE UNIVERSITY UNIVERSITY OF OREGON
UNIVERSITY OF SOUTHERN CALIFORNIA STANFORD UNIVERSITY UNIVERSITY OF HAWAII UNIVERSITY OF TOKYO UNIVERSITY OF UTAH WASHINGTON STATE UNIVERSITY UNIVERSITY OF WASHINGTON 


\section{Pacific Journal of Mathematics}

\section{Vol. 84 , No. 1 \\ May, 1979}

Michael James Beeson, Goodman's theorem and beyond ...............

Robert S. Cahn and Michael E. Taylor, Asymptotic behavior of multiplicities

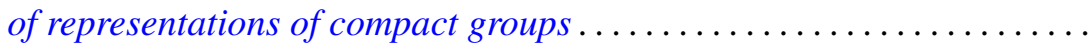

Douglas Michael Campbell and Vikramaditya Singh, Valence properties of

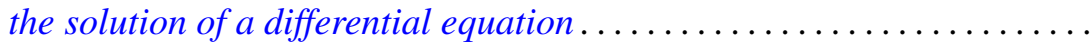

J.-F. Colombeau, Reinhold Meise and Bernard Perrot, A density result in spaces of Silva holomorphic mappings .....................

Marcel Erné, On the relativization of chain topologies .................

Le Baron O. Ferguson, Uniform and $L_{p}$ approximation for generalized

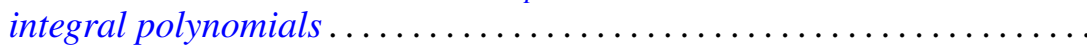

Kenneth R. Goodearl and David E. Handelman, Homogenization of regular rings of bounded index..................................

Friedrich Haslinger, A dual relationship between generalized

Abel-Gončarov bases and certain Pincherle bases .................

Miriam Hausman, Generalization of a theorem of Landau. .

Makoto Hayashi, 2-factorization in finite groups

Robert Marcus, Stochastic diffusion on an unbounded domain ........... 143

Isabel Dotti de Miatello, Extension of actions on Stiefel manifolds....

C. David (Carl) Minda, The hyperbolic metric and coverings of Riemann surfaces...

Somashekhar Amrith Naimpally and Mohan Lal Tikoo, On $T_{1}$-compactifications.

Chia-Ven Pao, Asymptotic stability and nonexistence of global solution for a semilinear parabolic equation ....

Shigeo Segawa, Harmonic majoration of quasibounded type ...

Sze-Kai Tsui and Steve Wright, The splitting of operator algebras ...

Bruce Williams, Hopf invariants, localization and embeddings of Poincaré complexes....

Leslie Wilson, Nonopenness of the set of Thom-Boardman maps ...

Alicia B. Winslow, There are $2^{\mathrm{c}}$ nonhomeomorphic continua in $\beta R^{n}-R^{n}$ 